\title{
Tue Gutes und rede darüber
}

\section{Remo Osterwalder}

Dr. med., Mitglied des Zentralvorstandes der FMH, Departementsverantwortlicher Freipraktizierende Ärztinnen und Ärzte

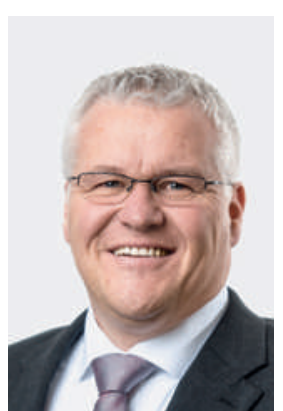

Was lange reift, wird endlich gut. Im Juni dieses Jahres konnte die neue operative Einheit des Departements Freipraktizierende Ärztinnen und Ärzte innerhalb des Generalsekretariates der FMH die Arbeit aufnehmen. Gerade in der aktuellen Zeit ist eine Situationsanalyse der Bedürfnisse der freipraktizierenden Ärzteschaft von enormer Bedeutung, insbesondere wenn es um Diskussionen der Praxisnachfolge sowie um die Anpassungen der Versorgungsmodelle geht, welche sich nach den Anliegen der zukünftigen Ärzteschaft richten sollten. Die neuen Ressourcen ermöglichten es, die ursprünglich von der Delegiertenversammlung in Auftrag gegebene Arbeitsgruppe (AG) «ambulante Basisversorgung" wieder zu reaktivieren. Durch diese AG sollen die Anliegen und Bedürfnisse der freipraktizierenden Ärzteschaft erfasst werden, um entsprechende Projekte optimal danach auszurichten. Aktuell befasst sich die AG mit den Themen des Praxiswildwuchses, der Generation Y und der innovativen Versorgungsmodelle.

\section{Praxiswildwuchs - welche Auswirkungen bringt er mit sich?}

Wir wollen uns auch in Zukunft für die therapeutische und wirtschaftliche Unabhängigkeit der freipraktizierenden Ärzteschaft einsetzen, denn einerseits sind die

Wer heute nicht weiss, wo er steht, kann morgen auch nicht sagen, wo er hingeht.

Tendenzen zu einem steigenden nicht-ärztlichen Einfluss im Gesundheitswesen stark bemerkbar. Andererseits ist die Versorgungsform der Einzelpraxis zunehmenden Veränderungen ausgesetzt: Immer häufiger werden Praxen nach der Pension des ehemaligen Inhabers an Praxisketten oder Netzwerke verkauft. Auch aus Sicht des Patienten ist es von zentraler Wichtigkeit, dass keine Interessenskonflikte in solchen Praxen bestehen. Da die Transparenz jedoch nicht standardisiert ist, ist es für den Patienten praktisch unmöglich, dies zu beurteilen.

\section{Die Generation Y - ein Begriff in aller Munde}

Wer heute nicht im Abseits stehen will, sollte sich mit der Generation Y auseinandersetzen. Flexibilität ist gefragt und wer die Lage richtig analysiert, hat einen wesentlichen Wettbewerbsvorteil. Das Anwerben und

\section{Nur wer die Zukunft aktiv mitgestaltet,} kann sich auch irren.

Halten dieser Mitarbeiter bilden kritische Faktoren für den wirtschaftlichen Erfolg eines Krankenhauses oder einer Arztpraxis [1]. Daher sind Kenntnisse von deren Ansprüchen sowie die entsprechenden Massnahmen von erheblicher Bedeutung. Infolgedessen schiessen Studien über die Generation Y wie Pilze aus dem Boden. Für die ältere Ärztegeneration gäbe es vieles zu lesen und zu lernen über die Nachfolge-Generation doch wie valide und reliabel sind diese Studien? $\mathrm{Zu}$ dem wird über die Generation Y meist nur in der Gesamtgeneration gesprochen. Die FMH interessiert sich jedoch für die Charakteristika der Ärzte der Generation Y - denn nur wer die Bedürfnisse dieser speziellen Zielgruppe genau kennt, kann adäquat darauf reagieren. Daher investieren wir einen wesentlichen Teil der Arbeit in das Projekt Generation Y.

\section{Versorgungsmodelle - welche sind erfolgreich?}

Unsere Abteilung ist der Meinung, dass die Entwicklung innovativer Versorgungsmodelle unterstützt und gefördert werden muss. Es soll in einem ersten Schritt eine Übersicht über die bereits existierenden, erfolgreichen Modelle geschaffen werden. Es gibt nicht nur «das» Modell, sondern so heterogen unsere Patientinnen und Patienten sind, so individuell sind die Bedürfnisse und Anforderungen an solche regionalen Modelle. Bestehende Strukturen wie Informations-Plattformen sollten gefördert werden. 